\title{
Mass Loss and Penetration Depth of Hypervelocity Projectiles
}

\author{
Tianhan Xu $\mathbb{D}^{1}{ }^{1}$ Yanyu Qiu, ${ }^{1,2}$ Jie Li $\mathbb{D}^{1},{ }^{1}$ Yihao Cheng, ${ }^{1}$ and Wei Chen ${ }^{3}$ \\ ${ }^{1}$ State Key Laboratory of Explosion \& Impact and Disaster Prevention \& Mitigation, Army Engineering University of PLA, \\ Nanjing 210007, China \\ ${ }^{2}$ School of Mechanical Engineering, Nanjing University of Science and Technology, Nanjing 210094, China \\ ${ }^{3}$ Engineering Quality Supervision Center of Logistics Support Department, PLA, Beijing 100048, China
}

Correspondence should be addressed to Jie Li; lijierf@163.com

Received 17 April 2021; Revised 22 June 2021; Accepted 11 July 2021; Published 23 July 2021

Academic Editor: Jinhua Li

Copyright (c) 2021 Tianhan $\mathrm{Xu}$ et al. This is an open access article distributed under the Creative Commons Attribution License, which permits unrestricted use, distribution, and reproduction in any medium, provided the original work is properly cited.

\begin{abstract}
Numerous investigations recorded a dramatic decrease in penetration depth at hypervelocity. The decrease is due to the severe mass loss of the projectile. The mechanics of mass loss are complicated, so the entirely theoretical model is still absent. In this paper, we derive a semitheoretical formula for mass loss by solving the equation of motion of the projectile during penetration. The result shows that the decrease in projectile mass at hypervelocity accords with the power law. Furthermore, we obtain a continuous formula for depth of penetration in the entire velocity domain. The theoretical results agree well with experimental data. The formula for the critical velocity of depth decreasing is also obtained as a by-product of the solving procedure.
\end{abstract}

\section{Introduction}

With the rapid development of hypervelocity air vehicles, the hitting speed of hypervelocity weapons has reached Mach 5-15 (Mach 1=340.3 m/s). There will be intense interactions on the interface between the projectile and the target at such high velocities. Melting, blunting, and mass loss of the projectile will occur. These phenomena weaken the projectile and diminish the terminal depth of penetration (DOP).

Numerous experiments have confirmed that projectile mass decreases dramatically under hypervelocity penetration. Forrestal and Piekutowski [1] used steel projectiles to penetrate aluminum targets and recorded the vanishing of the projectile at the impact velocity of $3075 \mathrm{~m} / \mathrm{s}$ with the help of radiographs. Qian et al. [2] conducted penetration experiments with tungsten alloy projectiles and concrete targets. The projectile was entirely eroded at the impact velocity of $3360 \mathrm{~m} / \mathrm{s}$. Li et al. [3] penetrated granite targets with alloy steel projectiles. The percentage of mass loss reached $88 \%$ when the impact velocity was $2378 \mathrm{~m} / \mathrm{s}$, and the velocity range of severe mass loss corresponded to that of DOP decreasing.
Projectile mass loss plays an essential role during the penetration and dominates the DOP in a specific range of velocity. Researchers have conducted numerous investigations on the mechanisms of mass loss. Silling and Forrestal [4] found a linear relationship between mass loss and the initial kinetic energy of projectiles. Klepaczko and Hughes [5] analyzed the wear at high sliding speeds and defined universal parameters to measure the wear intensity during penetration. Jones et al. [6] studied the work and heat during penetration and calculated the mass loss caused by surface melting. It is clear that many physical processes participate in the penetration process, leading to projectile mass loss. $\mathrm{He}$ et al. [7] combined several models and provided a formula with seven variables to estimate the mass loss.

These investigations were conducted on the condition that the impact velocity is below the transition velocity of semihydrodynamics, which is about $1000-1300 \mathrm{~m} / \mathrm{s}$. In this region, the DOP increases with impact velocity, though there is mass loss during penetration, and the projectiles maintain their primary forms after penetration. However, at hypervelocities, the dramatic decrease in DOP along with intense abrasion of projectiles will occur. It is evident that more complex physical processes occur during hypervelocity 
penetration, resulting in considerable difficulties in establishing accurate models to describe the process of mass loss. For engineering applications, a solution is to develop a semitheoretical model with unknown coefficients and to determine the coefficients by fitting experimental data.

In this paper, we analyze the penetration process of a cylindrical long rod projectile into a semi-infinite rock target and establish an equation of motion of the projectile. By solving the equation, we derive a formula for intense mass loss with one unknown coefficient. We further obtain the equations for DOP from low velocities to hypervelocities. Indepth analyses of the coefficients are carried out, and their physical meanings are clarified.

\section{Dynamic Behaviors of Projectile and Target during Penetration}

Figure 1 depicts the change of DOP with the impact velocity [3] ( $h$ is the DOP, $L$ is the total length of the projectile, and $v_{0}$ is the impact velocity). As shown in the figure, when the impact velocity is low, the DOP increases until the velocity reaches a critical value and then decreases dramatically with the velocity. After reaching the minimum, the DOP increases again with the velocity increasing. The change of the DOP is closely related to the states of projectile and target. Therefore, it is necessary to understand the behaviors of projectile and target during penetration under different impact velocities.

2.1. Dynamic Behaviors of the Projectile. With increasing the impact velocity, the interactions between projectile and target become more intense, and the behaviors of the projectile can be divided into four states as follows [3]:

(a) Rigid State. When the impact velocity is relatively low, the deformation and mass loss of the projectile are negligible, and the projectile can be regarded as a rigid body.

(b) Deformation State. When the pressure on the interface between projectile and target reaches the dynamic yield stress of the projectile, the projectile nose turns to the plastic state. Melting and mass loss also occur within a thin layer on the surface of the projectile nose. These factors blunt the projectile nose and slightly weaken the penetration efficiency of the projectile.

(c) Erosion State. With increasing the impact velocity, the interactions on the interface between projectile and target become more intense. The scope of yielding, melting, and mass loss of the projectile expands, leading to the failure of the structure of the projectile. In this state, the terminal DOP decreases with the impact velocity increasing.

(d) Hydrodynamic State. When the impact velocity further increases, the projectile as a whole enters the plastic flow state and can be regarded as a steadystate fluid. The projectile mass is entirely lost at the end of the penetration.

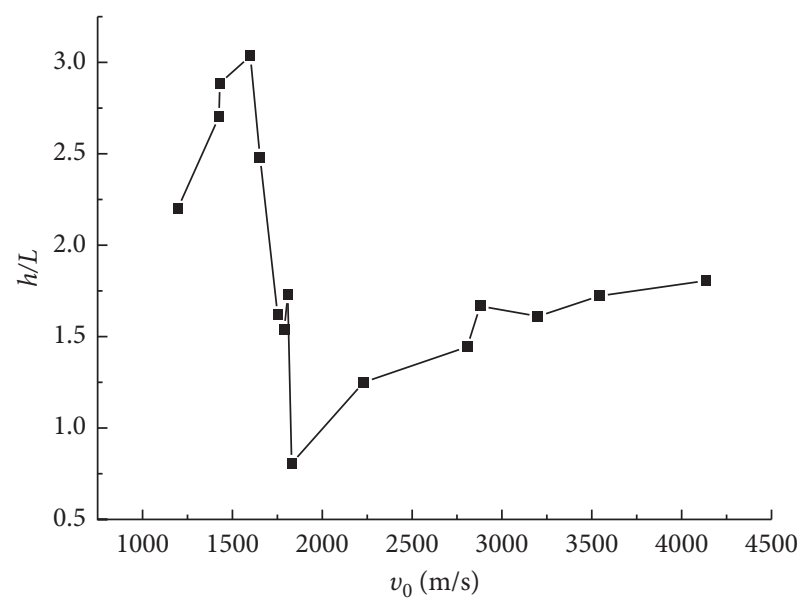

Figure 1: The curve of DOP vs. impact velocities.

2.2. Dynamic Behaviors of the Target. With the increase in the impact velocity, the pressure on the target side of the interface also increases. The stress-strain state of the target in the near-penetration region can be seen as the one-dimensional strain state, and the induced stress waves are onedimensional plane waves [8]. Metal targets turn to plastic flow at the elastic limit. Concrete and rock targets, however, turn to a hardening state at the elastic limit, and the hardening is caused due to internal friction [9]. The target with internal friction can be modeled by a solid that consists of small spheres with cohesion and friction. The "spheres" here refer to the groups of crystals with strong mutual bonds in real rocks. Since the target is in the state of one-dimensional strain under strong impact, we can consider a model solid in a cylinder with rigid walls, where compression occurs only along the cylinder axis. With the increase in compression, the solid undergoes three states in sequence:

(a) Elastic State. When the axial compression is small, friction is great, and cohesion is not broken. The deformation of the target obeys Hooke's law.

(b) Internal Friction State. When the axial compression reaches the elastic limit, cohesion is broken, and spheres slide on each other. Friction in this state is not negligible, and, therefore, penetration resistance of the projectile contains the term of internal friction.

(c) Hydrodynamic State. In this state, friction is negligible compared with great axial compression. The target can be considered as plastic fluid. The hydrodynamic term predominates in penetration resistance.

As we can see from the above analysis, both the projectile and the target undergo complex solid and fluid dynamic processes at hypervelocities. The terminal DOP is the result of the combination of various factors, which are not only involved in complex physical processes, but also affected by the randomness caused by the material inhomogeneity. It is difficult to establish an accurate model to predict the DOP 
for this condition, and it is unnecessary to do so for engineering applications. The appropriate approach is to analyze the factor that dominates the DOP and to establish a semitheoretical model.

\section{Mechanisms of the Decrease in DOP}

3.1. Influence of Projectile Yielding. Numerous experiments showed that when impact velocity reaches a critical value, the DOP does not increase with the velocity but decreases instead $[1-3,10,11]$. It is generally acknowledged that metal turns to plastic flow at the elastic limit. In this connection, the decrease in DOP may be due to the projectile yielding. If so, we can conveniently obtain the critical value of the impact velocity and the maximum DOP before decreasing.

We assume that the elastic limit of the projectile is larger than that of the target, and thus, the target turns to plastic before the projectile. When the projectile is still elastic, the pressure on the interface at the instant of impact is

$$
p=\rho_{p} c_{p} v_{L}=\rho_{t} D_{t} v_{t},
$$

where $\rho_{p}$ and $\rho_{t}$ are, respectively, densities of the projectile and the target, $D_{t}$ is the shock wave velocity of the target, $c_{p}$ is the elastic longitudinal wave velocity of the projectile, and $v_{L}$ and $v_{t}$ are, respectively, particle velocities of the projectile and the target on the interface.

The shock wave velocity $D_{t}$ is usually written as

$$
D_{t}=a_{1}+a_{2} v_{t},
$$

where $a_{1}$ and $a_{2}$ are coefficients related to materials and can be determined by fitting experimental data.

Shemyakin [9] investigated the behavior of rock under dynamic loadings and concluded that the wave velocity in the vicinity of the loading source is approximately equal to elastic wave velocity, i.e. $D_{t} \approx c_{t}$, where $c_{t}$ is the elastic longitudinal wave velocity of the target.

Assuming that the initial particle velocity of the target equals the impact velocity, we can give the condition where the projectile yields, i.e.,

$$
p=\rho_{t} D_{t} v_{y} \approx \rho_{t} c_{t} v_{y}=\sigma_{y p},
$$

where $v_{y}$ is the minimum impact velocity at which the projectile yields and $\sigma_{y p}$ is the dynamic compression strength of the projectile.

Whiffin [12] conducted Taylor impact tests with mild steel projectiles of different sizes and confirmed that the dynamic compression strength of projectiles is constant (see Figure 2).

Besides, Whiffin [12] obtained the relationships between the static and dynamic compression strength of steel and alloy, i.e.,

$$
\frac{\sigma_{y}}{\sigma_{s}}=8.84-2.42 \lg \sigma_{s}
$$

for steel and

$$
\frac{\sigma_{y}}{\sigma_{s}}=6.32-1.89 \lg \sigma_{s}
$$

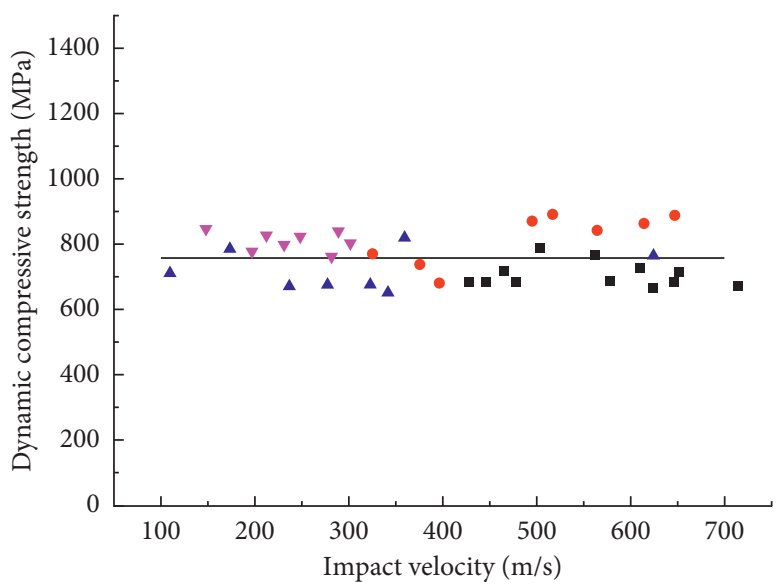

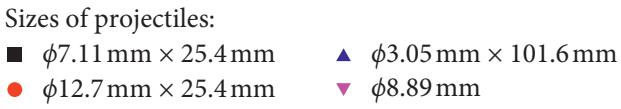

FIgURE 2: Dynamic compression strength of projectiles at different impact velocities.

for aluminum alloy, where $\sigma_{s}(\mathrm{MPa})$ and $\sigma_{y}(\mathrm{MPa})$ are static and dynamic compression strengths, respectively.

We calculate $v_{y}$ with different experimental data, and the results are listed in Table 1, where $\sigma_{s p}$ is the static compression strength of the projectile, and $v_{m}$ is the critical velocity of decrease in DOP.

As shown in Table 1, velocities at which projectiles yield are much smaller than those at which DOPs decrease, indicating that projectiles have yielded before DOP decreasing. It is also worth noting that the critical velocities are nonlinearly related to the strength of the projectile and target. The analysis above confirms that the decrease in DOP is due to the combination of different factors.

3.2. Dominant Mechanism of the Decrease in DOP. Existing investigations confirmed that blunting and mass loss of projectiles weaken the penetration efficiency of projectiles $[4,7,13-17]$, provided that impact velocities are less than the critical velocities. In this subsection, we analyze the influences of blunting and mass loss at hypervelocities, aiming to figure out the dominant mechanism of the decrease in DOP.

Figure 3 depicts the variation of caliber-radius-head $(\mathrm{CRH})$ and DOP with the impact velocity, where $f_{c}$ is the axial compression strength of targets. It is evident that the $\mathrm{CRH}$ value of the posttest projectiles decreases with the impact velocity and approaches a limit of 0.5 , which represents a hemispherical projectile nose. Chen et al. [19] indicated that the limit of $\mathrm{CRH}$ defines a theoretical limit of projectile mass loss. As shown in Figure 3, DOP keeps increasing with the velocity after $\mathrm{CRH}$ reaches its limit. We can thus infer that the blunting of the projectile nose contributes little to the decrease in DOP.

Figure 4 shows the variation of the ratio of posttest projectile mass with the impact velocity. As shown in the figure, with increasing the impact velocity, the residual projectile mass decreases slowly at first and then drops 
TABLE 1: Parameters of projectiles and targets in different penetration tests.

\begin{tabular}{|c|c|c|c|c|c|c|c|}
\hline Data sources & $\sigma_{s p}(\mathrm{MPa})$ & $\sigma_{y p}(\mathrm{MPa})$ & $\rho_{t}\left(\mathrm{~kg} / \mathrm{m}^{3}\right)$ & $c_{t}(\mathrm{~m} / \mathrm{s})$ & $v_{y}(\mathrm{~m} / \mathrm{s})$ & $v_{m}(\mathrm{~m} / \mathrm{s})$ & $v_{m} / v_{y}$ \\
\hline \multirow{3}{*}{ Forrestal and Piekutowski [1] } & 1115 & 1634 & 2700 & 5000 & 121 & 892 & 7.4 \\
\hline & 1245 & 1680 & 2700 & 5000 & 124 & 932 & 7.5 \\
\hline & 1490 & 1730 & 2700 & 5000 & 128 & 1086 & 8.5 \\
\hline Li et al. [3] & 1952 & 1952 & 2670 & 4200 & 174 & 1600 & 9.2 \\
\hline Qian et al. [2] & 731 & 1396 & 2200 & 4000 & 159 & 2600 & 16.4 \\
\hline
\end{tabular}

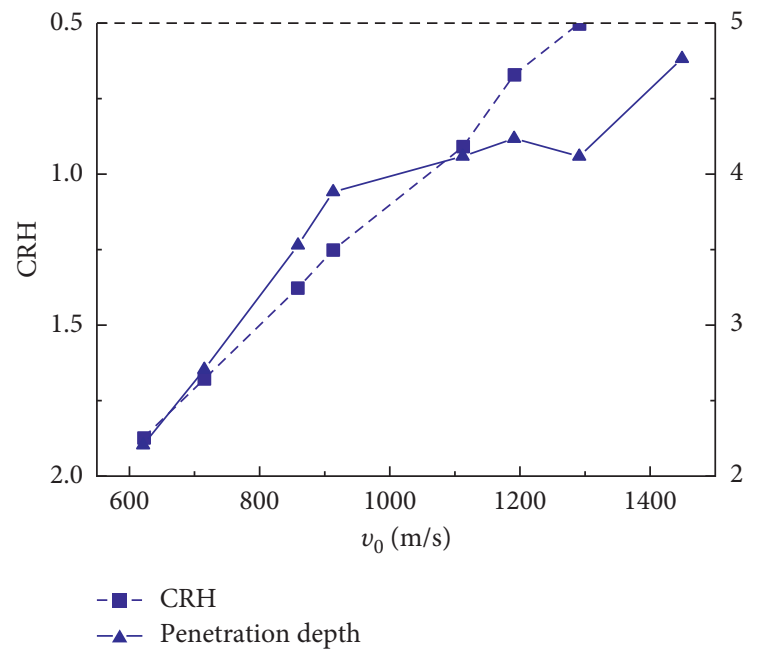

(a)

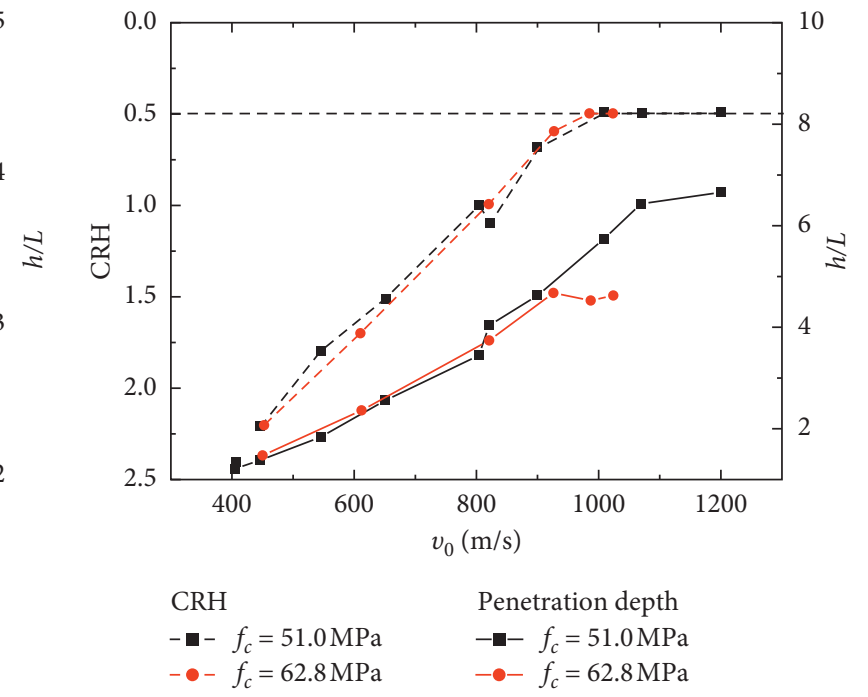

(b)

Figure 3: Curves of CRH and DOP vs. impact velocity. (a) Data from reference [18]. (b) Data from reference [10].

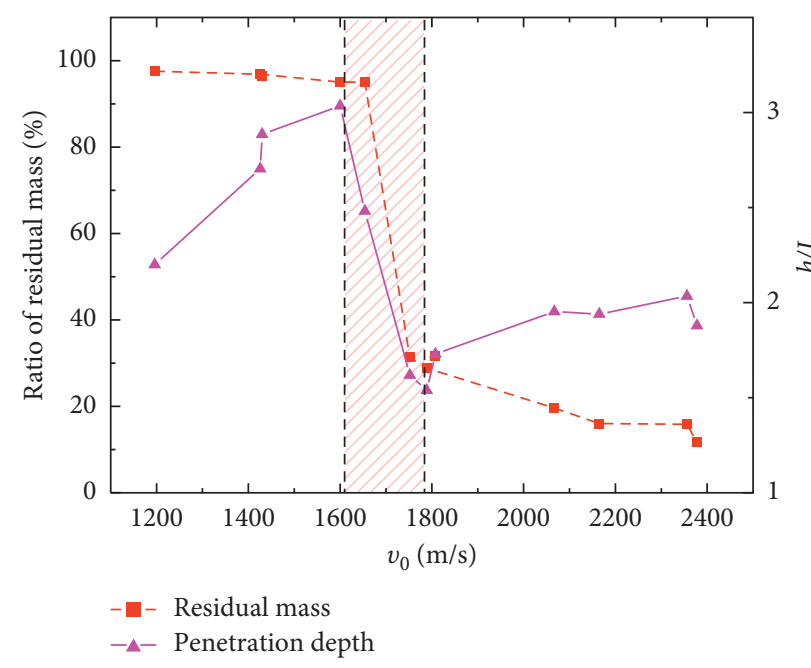

(a)

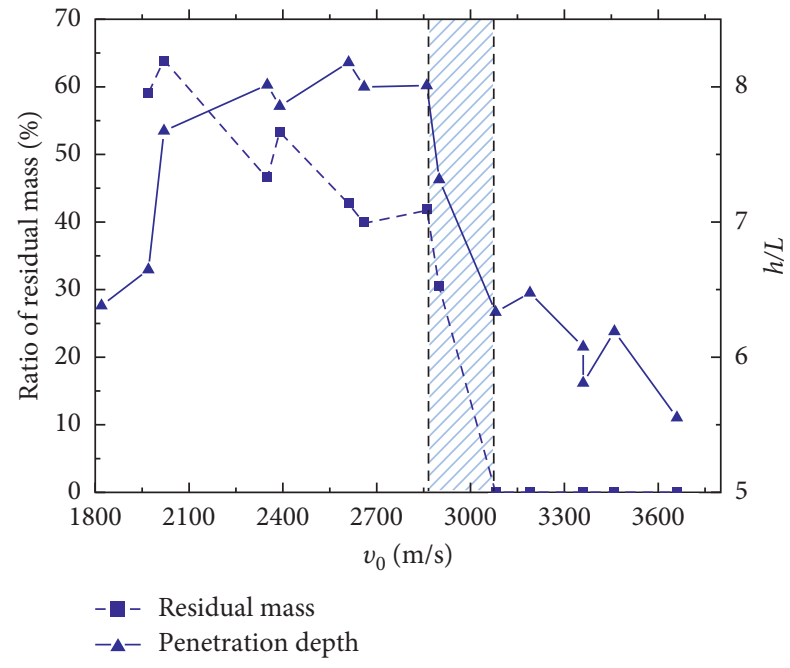

(b)

Figure 4: Curves of DOP and the ratio of residual mass vs. impact velocity. (a) Data from reference [20]. (b) Data from reference [2].

rapidly at a critical value of the velocity. In Figure 4(a), the ratio of residual mass decreases from $90 \%$ to $20 \%$ when the impact velocity increases from $1600 \mathrm{~m} / \mathrm{s}$ to $1800 \mathrm{~m} / \mathrm{s}$. In Figure 4 (b), the ratio of residual mass decreases from $42 \%$ to nearly 0 when the impact velocity increases from $2860 \mathrm{~m} / \mathrm{s}$ to $3080 \mathrm{~m} / \mathrm{s}$. It is worth noting that the velocity region in which DOP decreases is consistent with that in which the residual mass decreases dramatically (the region is stressed by a 
hatched pattern in the figure). This consistency indicates that the decrease in DOP is due to the severe mass loss of the projectile.

Figure 5 shows six posttest radiographs of targets and projectiles from Reference [1], and the corresponding DOPs are depicted in Figure 6 by points A-F, respectively. The radiographs with $v_{0}=781 \mathrm{~m} / \mathrm{s}$ and $v_{0}=932 \mathrm{~m} / \mathrm{s}$ show slight bending and blunting of the projectiles. Points $\mathrm{A}$ and $\mathrm{B}$ in Figure 6 indicate DOP increasing in this velocity region. When the velocity increases to $1037 \mathrm{~m} / \mathrm{s}$ and $1193 \mathrm{~m} / \mathrm{s}$, large bending and shortening of the projectiles occur, leading to a dramatic decrease in DOP, as shown by points $\mathrm{C}$ and $\mathrm{D}$ in Figure 6 . The surface of the projectile is extremely rough at $v_{0}=1193 \mathrm{~m} / \mathrm{s}$, which implies severe abrasion and mass loss of the projectile. At the velocities of $1802 \mathrm{~m} / \mathrm{s}$ and $3075 \mathrm{~m} / \mathrm{s}$, abrasion and mass loss are more intense, the projectile bodies are broken, and the DOP increases again with the velocity increasing.

Recalling the dynamic behaviors of the projectile discussed in Subsection 2.1, we can now analyze the mechanics of DOP in different velocity ranges. In the rigid and deformation states, although yielding, blunting, and slight mass loss of the projectile have occurred, DOP still increases with the velocity. This is because the increase in the impact velocity compensates for the decrease in DOP caused by mass loss and blunting of the projectile. In this region, the projectile can be seen as a rigid body, since the DOP increases linearly with the velocity. With increasing the impact velocity, the scopes of abrasion, yielding, and melting expand. At a critical velocity, the projectile loses the capacity to maintain its primary form, and severe mass loss occurs. In this state, i.e., abrasion state, the increase in the impact velocity can no longer compensate for the decrease in DOP caused by mass loss; thus, DOP decreases dramatically with the velocity increasing. With a further increase in the velocity, the projectile turns to the hydrodynamic state and can be regarded as a steady-state flow. DOP increases again with the velocity increasing, because the residual mass is so small that the effect of mass loss is little in this region.

\section{Theoretical Model of Mass Loss}

To analyze the motion of projectiles in the erosion stage, we study the process of a projectile penetrating vertically into a semi-infinite target. As stated in Subsection 3.2, the shape of the projectile nose has changed to a hemisphere when severe mass loss happens. Therefore, the original nose shape has little effect on the DOP in this stage. In this connection, we assume that a cylindrical projectile impacts the target orthogonally with the initial velocity $v_{0}$, taking the initial static target as the reference frame. During the penetration, the instant velocity of the projectile tail is $v_{\mathrm{p}}$ and the instant particle velocity of the target on the interface is $v_{t}$, as shown in Figure 7.

The particle velocity of the projectile nose relative to the projectile tail is

$$
v_{L}=v_{p}-v_{t}
$$

On the interface of projectile and target, considering that shock wave velocities approximately equal to elastic wave velocities, we have [21]

$$
\rho_{p} c_{p} v_{L}=\rho_{t} c_{t} v_{t}
$$

Combining equations (6) and (7) yields

$$
\begin{aligned}
& v_{L}=\frac{v_{p}}{1+\left(\rho_{p} c_{p} / \rho_{t} c_{t}\right)}, \\
& v_{t}=\frac{v_{p}}{1+\left(\rho_{t} c_{t} / \rho_{p} c_{p}\right)} .
\end{aligned}
$$
as [20]

The resistance force against penetration can be expressed

$$
\sigma_{c}=\alpha_{s}+\beta_{s} v_{t},
$$

where $\alpha_{s}$ and $\beta_{s}$ are coefficients, and $\sigma_{c}$ is resistance force per unit area on the cross-section of the projectile. At medium and high velocities, we can prove that $\beta_{s} v_{t} \gg \alpha_{s}$; thus, $\alpha_{s}$ is negligible.

When the initial impact velocity, $v_{0}$, exceeds the critical velocity, $v_{m}$, the projectile enters the erosion state, where the equation of motion of the projectile is

$$
-\sigma_{c} A_{0}=\rho_{p} A_{0} l \frac{\mathrm{d} v_{p}}{\mathrm{~d} t},
$$

where $A_{0}$ is the cross-section area of the projectile, and $l$ is the instant length of the entire residual projectile during the penetration. In fact, the particle velocity $v_{L}$ equals the rate of projectile shortening, i.e., $\mathrm{d} l / \mathrm{d} t=-v_{L}$.Equation (10) can thus be written as

$$
\beta_{s} v_{t} A_{0} \frac{\mathrm{d} l}{l}=\rho_{p} A_{0} v_{L} \mathrm{~d} v_{p} .
$$

Substituting equation (8) into equation (11) and integrating both sides yield

$$
l=C \exp \left(\frac{\rho_{p}}{\beta_{s}} \frac{Z_{2}}{Z_{1}} v_{p}\right),
$$

where $C$ is the undetermined coefficient, $Z_{1}=1+$ $\left(\rho_{p} c_{p} / \rho_{t} c_{t}\right)$, and $Z_{2}=1+\left(\rho_{t} c_{t} / \rho_{p} c_{p}\right)$.

Taking into account the initial condition that $\left.v_{p}\right|_{l=L}=v_{0}$, we have

$$
\frac{l}{L}=\exp \left[\frac{\rho_{p}}{\beta_{s}} \frac{Z_{2}}{Z_{1}}\left(v_{p}-v_{0}\right)\right] .
$$

It follows from equation (13) that the instant mass of the projectile, $m$, is

$$
\frac{m}{m_{0}}=\exp \left[\frac{\rho_{p}}{\beta_{s}} \frac{Z_{2}}{Z_{1}}\left(v_{p}-v_{0}\right)\right],
$$

provided that $l / L=m / m_{0}$, where $m_{0}$ is the initial mass of the projectile.

Equation (14) can be recast as 


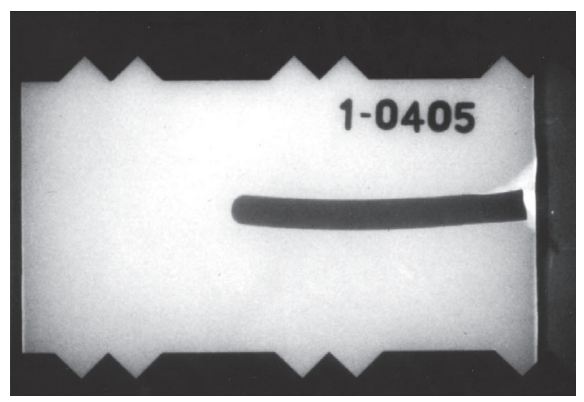

(a)

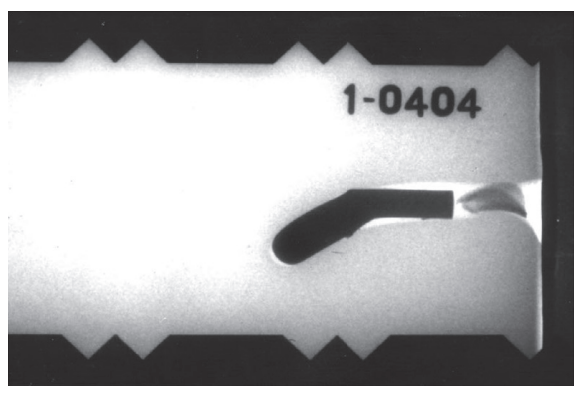

(c)

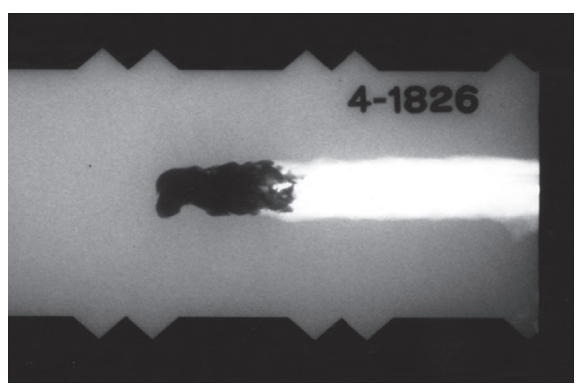

(e)

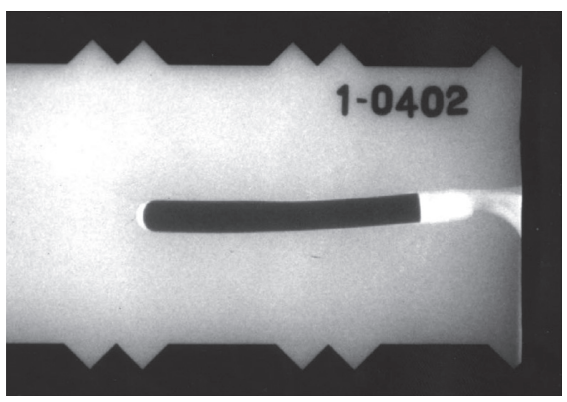

(b)

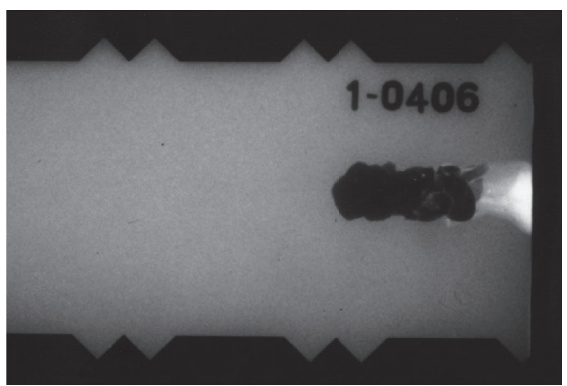

(d)

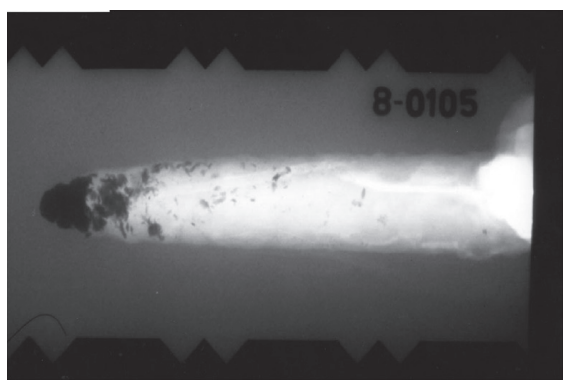

(f)

Figure 5: Posttest radiographs of targets and projectiles. (a) $781 \mathrm{~m} / \mathrm{s}$. (b) $932 \mathrm{~m} / \mathrm{s}$. (c) $1037 \mathrm{~m} / \mathrm{s}$. (d) $1193 \mathrm{~m} / \mathrm{s}$. (e) $1802 \mathrm{~m} / \mathrm{s}$. (f) $3075 \mathrm{~m} / \mathrm{s}$.

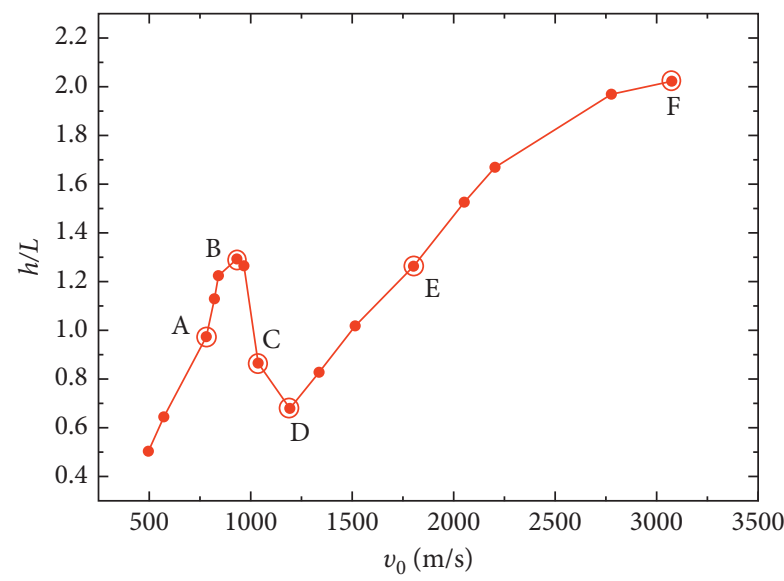

Figure 6: The curve of DOP vs. the impact velocity.

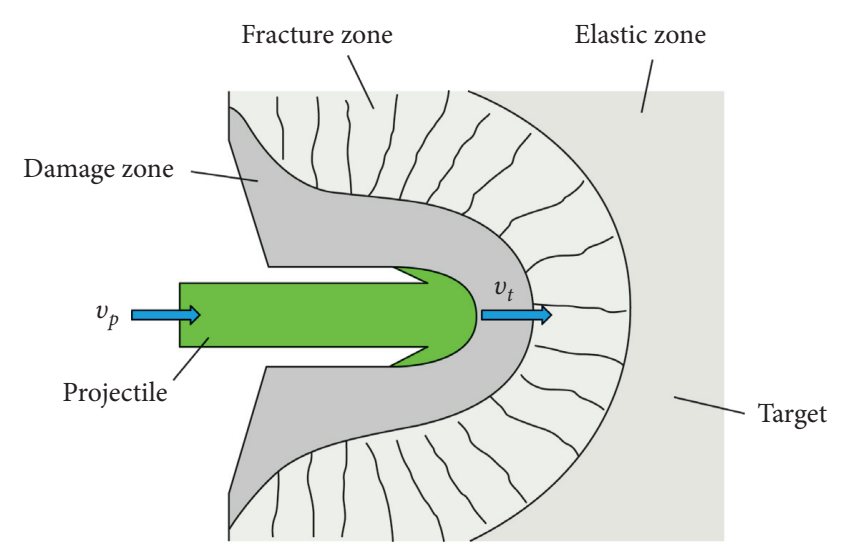

Figure 7: Schematic diagram of the projectile penetrating the target. 


$$
m=m_{0} \exp \left[\alpha\left(\frac{v_{p}-v_{0}}{v_{m}}\right)\right]
$$

where $\alpha=\left(\rho_{p} / \beta_{s}\right)\left(Z_{2} / Z_{1}\right) v_{m}$.

The projectile turns to the rigid state when $v_{p}$ decreases to $v_{m}$, and thus, the residual mass of the projectile is

$$
m_{\mathrm{res}}=m_{0} \exp \left[\alpha\left(1-\frac{v_{0}}{v_{m}}\right)\right] \text {. }
$$

It follows from equations (15) and (16) that $\alpha$ is the rate of mass loss when $v_{0}>v_{\mathrm{m}}$. In the above solution procedure, $\alpha$ is determined by the penetration resistance and strengths of projectile and target. As we have known, however, there are at least six factors that have effects on mass loss.
Therefore, $\alpha$ can be regarded as an unknown coefficient that is determined by fitting experimental data.

\section{Calculation of DOP}

5.1. DOP of Rigid Penetration. When $v_{0} \leq v_{\mathrm{m}}$, the mass loss has little effect on DOP, and the projectile can be seen as a rigid body. For a rigid projectile, $v_{t}=v_{p}$, and thus, the equation of motion of the projectile can be expressed as

$$
-\sigma_{c} A_{0}=m_{0} \frac{\mathrm{d} v_{p}}{\mathrm{~d} t}=m_{0} \frac{\mathrm{d} v_{t}}{\mathrm{~d} t}=m_{0} \frac{\mathrm{d}^{2} h^{\prime}}{\mathrm{d} t^{2}},
$$

where $h^{\prime}$ is the instant DOP during the penetration process.

Substituting equation (9) into equation (17) yields the formula for DOP of rigid projectile:

$$
h=-\frac{m_{0}}{\beta_{s} A_{0}} \int_{v_{0}}^{0}\left(1-\frac{\alpha_{s}}{\alpha_{s}+\beta_{s} v_{0}}\right) \mathrm{d} v_{p}=\frac{m_{0}}{\beta_{s} A_{0}}\left[v_{0}-\left(\frac{\alpha_{s}}{\beta_{s}}\right) \ln \left(1+\left(\frac{\beta_{s}}{\alpha_{s}}\right) v_{0}\right)\right] \text {. }
$$

The coefficients $\alpha_{s}$ and $\beta_{s}$ are determined as follows [20]:

$$
\begin{aligned}
\alpha_{s} & =2 \tau_{s}, \\
\beta_{s} & =\rho_{t} c_{t} \lambda_{d} N_{2}, \\
\lambda_{d} & =1-1.12^{n}\left(\varepsilon_{0} \chi\right)^{n / 4}, \\
\chi & =\frac{r_{0}}{\Delta} \\
\Delta & =\left(\frac{K_{c}}{\tau_{s}}\right)^{2}, \\
\varepsilon_{0} & =\frac{\tau_{s}}{G}, \\
N_{2} & =\left(\frac{12 \psi^{2}-4 \psi+1}{6 \psi}\right) \sqrt{4 \psi-1}-2 \psi(2 \psi-1)\left(\frac{\pi}{2}-\theta_{0}\right), \\
\theta_{0} & =\arcsin (1-0.5 \psi),
\end{aligned}
$$

where $n=1.6-1.8, \varepsilon_{0}$ is the limit of the shear strain of the target, $\tau_{s}$ is the shear strength of the target, $G$ is the shear modulus of the target, $r_{0}$ is the projectile radius, $K_{c}$ is the fracture toughness of the projectile, and $\psi$ is the CRH value.

For rock targets, $\beta_{s} v_{0} \gg \alpha_{s}$ when $v_{0}>500 \mathrm{~m} / \mathrm{s}$, and thus, $\alpha_{s}$ is negligible $[3,20]$. Then, equation (18) can be simplified to

$$
h=\frac{m_{0}}{A_{0} \beta_{s}} v_{0}, \quad 0<v_{0} \leq v_{m}
$$

5.2. DOP of Erosion Penetration. When the impact velocity, $v_{0}$, exceeds the critical velocity, $v_{m}$, the projectile turns to the erosion state, where projectile mass and DOP decrease dramatically. Because the instant velocity, $v_{p}$, keeps decreasing during the penetration process, the DOP consists of two parts, i.e., the erosion part when $v_{p}>v_{m}$ and the rigid part when $v_{p} \leq v_{m}$.

For $v_{p}>v_{m}$, the equation of motion of the projectile can be expressed as

$$
m \frac{\mathrm{d} v_{p}}{\mathrm{~d} t}=-\beta_{s} v_{t} \pi r_{0}^{2}=-\beta_{s} \pi r_{0}^{2} \frac{\mathrm{d} h}{\mathrm{~d} t}
$$

Substituting equation (15) for the projectile mass in equation (21) yields

$$
m_{0} \exp \left[\alpha\left(\frac{v_{p}-v_{0}}{v_{m}}\right)\right] \mathrm{d} v_{p}=-\beta_{s} \pi r_{0}^{2} \mathrm{~d} h
$$

The velocity decreases from $v_{0}$ to $v_{m}$ while DOP increases from 0 to $h_{s 1}$; thus, the DOP of the erosion stage can be obtained by integrating equation (21), i.e.,

$$
h_{s 1}=\frac{m_{0} v_{m}}{\beta_{s} \pi r_{0}^{2} \alpha}\left\{1-\exp \left[\alpha\left(1-\frac{v_{0}}{v_{m}}\right)\right]\right\} \text {. }
$$

When $v_{p}$ decreases to $v_{m}$, the residual projectile keeps penetrating as a rigid body. This part is equivalent to the rigid penetration with the initial impact velocity $v_{m}$ and the projectile mass $m_{\text {res }}$. The DOP of this part can be obtained by substituting equation (16) for the projectile mass in equation (19), i.e.,

$$
h_{s 2}=\frac{m_{0} v_{m}}{\pi r_{0}^{2} \beta_{s}} \exp \left[\alpha\left(1-\frac{v_{0}}{v_{m}}\right)\right] \text {. }
$$

The terminal DOP is

$$
\begin{aligned}
& h_{s}=h_{s 1}+h_{s 2}=\lambda_{c} \frac{m_{0} v_{0}}{\beta_{s} \pi r_{0}^{2}}, \quad v_{0}>v_{m}, \\
& \lambda_{c}=\frac{v_{m}}{v_{0}} \frac{1}{\alpha}\left\{1+(\alpha-1) \exp \left[\alpha\left(1-\frac{v_{0}}{v_{m}}\right)\right]\right\},
\end{aligned}
$$


where $\lambda_{c}$ can be termed as "decrease coefficient." $h_{s}$ has the same form as equation (20). When $v_{0}>v_{m}, \lambda_{c}<1$, implying that DOP is less than that in the rigid state at the same impact velocity. In this connection, we can examine the validity of equation (16) by comparing theoretical and experimental results of DOP.

5.3. DOP of Hydrodynamic Penetration. In the hydrodynamic state, the behaviors of projectile and target can be described as steady-state fluids, and the modified Bernoulli equation applies, i.e., [3]

$$
\frac{1}{2} \rho_{p}\left(v_{p}-v_{t}\right)^{2}=H+\frac{\kappa}{2} \rho_{t} v_{t}^{2},
$$

where $H$ is the dynamic hardness of the target, and $\kappa$ is the coefficient describing the state of the target. $\kappa=1$ stands for the ideal liquid state of the target. The relationship between $\kappa$ and the impact velocity is determined by Boltzmann's function [3]:

$$
\kappa= \begin{cases}\kappa_{0}, & M_{*} \leq 1.5, \\ \frac{2 \kappa_{0}-1+\exp M_{*}^{\prime}}{1+\exp M_{*}^{\prime}}, & M_{*}>1.5,\end{cases}
$$

where $\quad M_{*}^{\prime}=\left(M_{*}-1.5\right) / 0.5, \quad M_{*}=v_{0} / c, \quad c=\sqrt{2 H / \rho_{t}}$, $\kappa_{0}=(1+\nu) /(3(1-v))$, and $\nu$ is Poisson's ratio of the target.

The DOP in the hydrodynamic state can be derived from equation (26), i.e.,

$$
h=L \lambda\left(\frac{\lambda-\theta}{\lambda \theta-\kappa}\right)
$$

where $\lambda=\sqrt{\rho_{p} / \rho_{t}}$ and $\theta=\sqrt{\kappa+\left(1-\kappa / \lambda^{2}\right) / M_{*}^{2}}$.

We can see that $\theta \longrightarrow \sqrt{\kappa}$ as $M_{*}$, i.e., $v_{0}$, increases. Besides, it follows from equation (27) that $\kappa \longrightarrow 1$ as $v_{0}$ increases. Equation (28) can thus be simplified into

$$
\frac{h}{L}=\lambda .
$$

Equation (29) is the DOP of ideal jet flow. The projectile length $L$ is equivalent to the length of the jet flow.

In equation (28), the whole projectile turns to the hydrodynamic state. As shown in Figures 5(a) and 5(b), however, the projectile, though a large proportion of which is lost, still remains at the end of hypervelocity penetration. In this connection, there is no clear boundary between the erosion state and the hydrodynamic state. To obtain the continuous calculation formula for DOP, we assume that the erosion part of the projectile keeps penetrating in the form of fluid.

The equivalent jet length of the erosion part is

$$
L^{\prime}=L \frac{m_{0}-m_{\mathrm{res}}}{m_{0}}=L\left\{1-\exp \left[\alpha\left(1-\frac{v_{0}}{v_{m}}\right)\right]\right\} \text {. }
$$

Substituting equation (30) into equation (28) yields the DOP of the jet flow, i.e.,

$$
h_{f}=L \lambda\left(\frac{\lambda-\theta}{\lambda \theta-\kappa}\right)\left\{1-\exp \left[\alpha\left(1-\frac{v_{0}}{v_{m}}\right)\right]\right\} .
$$

Recalling equation (25), the total DOP when $v_{0}>v_{m}$ is

$$
h=h_{s}+h_{f} \text {. }
$$

Equation (32) is valid for conditions, where impact velocities are greater than the critical velocity. The only unknown coefficient $\alpha$ can be determined by fitting experimental data. When the impact velocity is relatively low, $h_{s}$ dominates the terminal DOP; when the impact velocity is high, however, $h_{f}$ predominates.

5.4. Analysis of the Penetration Formulas. If we rewrite the initial projectile mass as

$$
m_{0}=\vartheta \rho_{p} \pi r_{0}^{2} L,
$$

where $\vartheta$ is the coefficient related to the geometry of the projectile, then the formulas of $h_{s 1}, h_{s 2}$, and $h_{f}$ can be expressed in normalized forms that are independent of projectile size, i.e.,

$$
\begin{aligned}
& \frac{h_{s 1}}{L}=9 \lambda^{2} \frac{v_{m}}{c_{t} \lambda_{d} N_{2} \alpha}\left\{1-\exp \left[\alpha\left(1-\frac{v_{0}}{v_{m}}\right)\right]\right\}, \\
& \frac{h_{s 2}}{L}=9 \lambda^{2} \frac{v_{m}}{c_{t} \lambda_{d} N_{2}} \exp \left[\alpha\left(1-\frac{v_{0}}{v_{m}}\right)\right], \\
& \frac{h_{f}}{L}=\lambda^{2}\left(\frac{1-\theta / \lambda}{\theta-\kappa / \lambda}\right)\left\{1-\exp \left[\alpha\left(1-\frac{v_{0}}{v_{m}}\right)\right]\right\} .
\end{aligned}
$$

It follows from equation (16) that

$$
\begin{aligned}
\frac{m_{\text {res }}}{m_{0}} & =\exp \left[\alpha\left(1-\frac{v_{0}}{v_{m}}\right)\right], \\
\frac{m_{0}-m_{\text {res }}}{m_{0}} & =1-\exp \left[\alpha\left(1-\frac{v_{0}}{v_{m}}\right)\right] .
\end{aligned}
$$

The left-hand sides of equation (37) represent the proportions of residual mass and erosion mass, respectively. Equations (33)-(35) can be recast as

$$
\begin{aligned}
& \frac{h_{s 1}}{L}=\left(\vartheta \lambda^{2} \frac{v_{m}}{c_{t} \lambda_{d} N_{2}}\right) \frac{m_{0}-m_{\mathrm{res}}}{m_{0}} \phi, \\
& \frac{h_{s 2}}{L}=\left(\vartheta \lambda^{2} \frac{v_{m}}{c_{t} \lambda_{d} N_{2}}\right) \frac{m_{\mathrm{res}}}{m_{0}}, \\
& \frac{h_{f}}{L}=\lambda^{2}\left(\frac{1-\theta / \lambda}{\theta-\kappa / \lambda}\right) \frac{m_{0}-m_{\mathrm{res}}}{m_{0}},
\end{aligned}
$$

where $\phi=1 / \alpha$. We can see that equations (38) and (39) are similar in form. Considering that equation (39) represents the DOP of a rigid projectile with the mass of $m_{\text {res }}$ and the initial velocity of $v_{m}$, while equation (38) represents the DOP of a projectile, whose mass is entirely lost just at the end of 


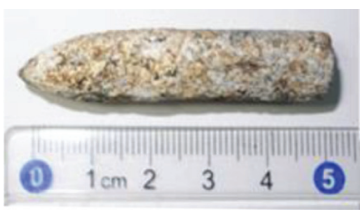

(a)

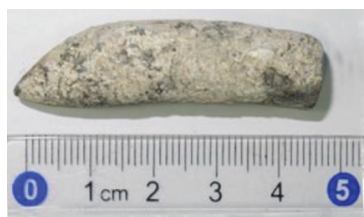

(e)

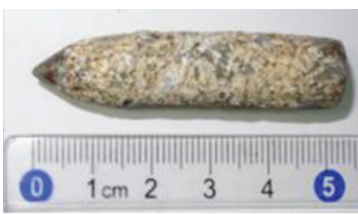

(b)

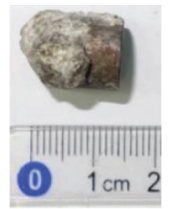

(f)

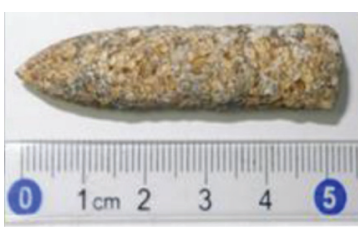

(c)

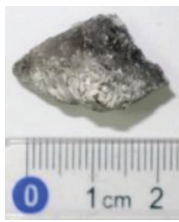

(g)

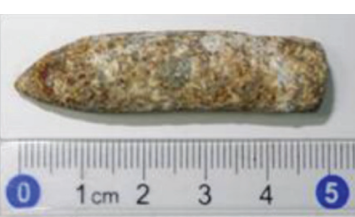

(d)

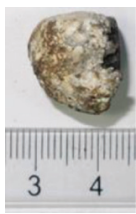

(h)

Figure 8: Posttest projectiles at different impact velocities. (a) $v_{0}=1196 \mathrm{~m} / \mathrm{s}$. (b) $v_{0}=1426 \mathrm{~m} / \mathrm{s}$. (c) $v_{0}=1430 \mathrm{~m} / \mathrm{s}$. (d) $v_{0}=1600 \mathrm{~m} / \mathrm{s}$. (e) $v_{0}=1654 \mathrm{~m} / \mathrm{s}$. (f) $v_{0}=1752 \mathrm{~m} / \mathrm{s}$. (g) $v_{0}=1789 \mathrm{~m} / \mathrm{s}$. (h) $v_{0}=1808 \mathrm{~m} / \mathrm{s}$.

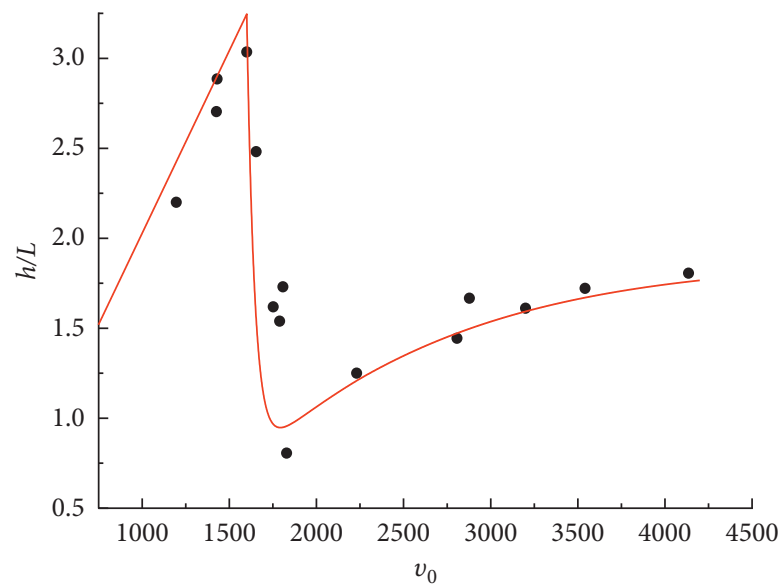

- Experimental data

- Theoretical results

(a)

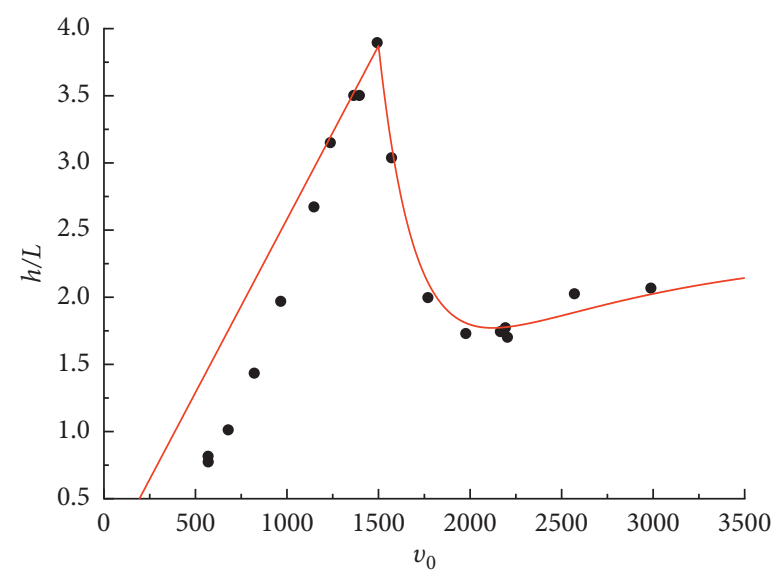

- Experimental data Theoretical results

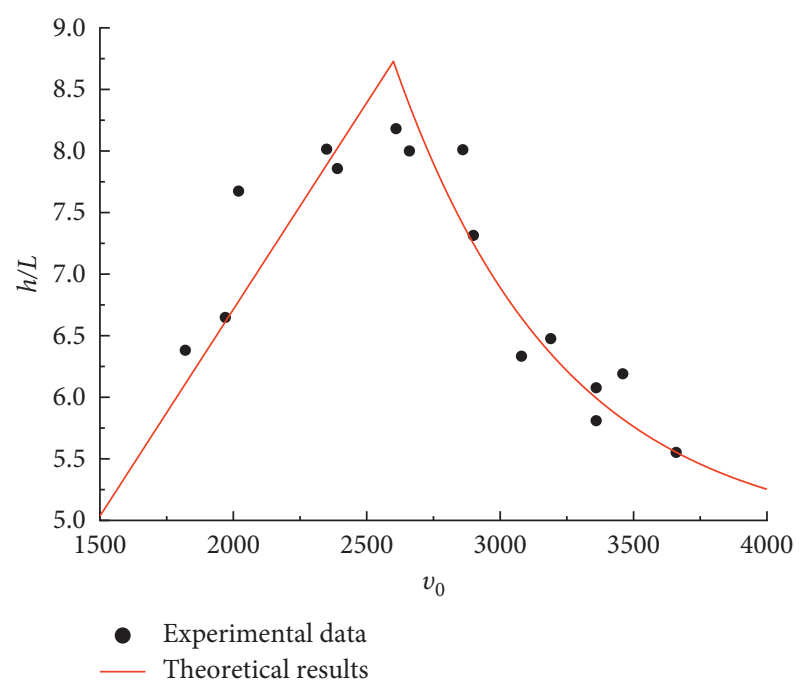

(b)

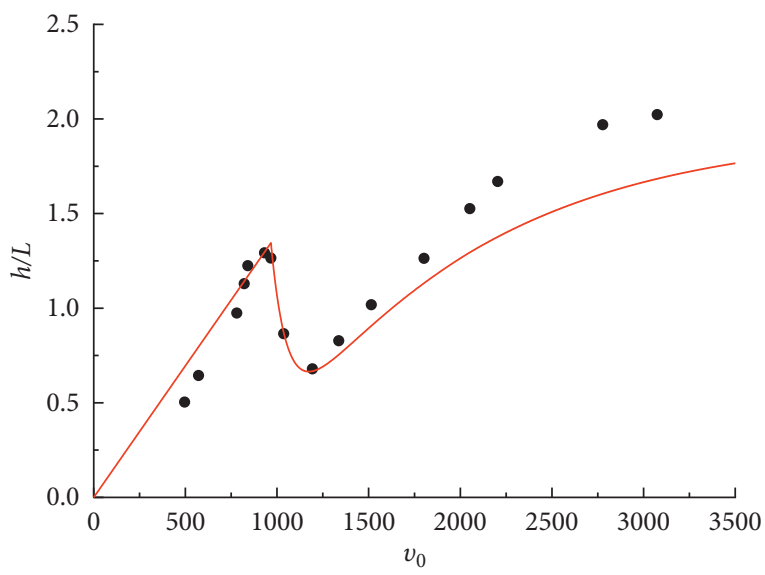

- Experimental data - Theoretical results

(c)

(d)

FIgURE 9: Theoretical and experimental DOPs of penetration experiments. (a) $v_{m}=1600 \mathrm{~m} / \mathrm{s}$ and $\alpha=35$. (b) $v_{m}=2600 \mathrm{~m} / \mathrm{s}$ and $\alpha=4.3$. (c) $v_{m}=1500 \mathrm{~m} / \mathrm{s}$ and $\alpha=7$. (d) $v_{m}=967 \mathrm{~m} / \mathrm{s}$ and $\alpha=10$. 
TABLe 2: Calculated $v_{m}$ with different experimental results.

\begin{tabular}{lccc}
\hline$v_{0}(\mathrm{~m} / \mathrm{s})$ & DOP $(\mathrm{cm})$ & $m_{\text {res }}(\mathrm{g})$ & $v_{m}(\mathrm{~m} / \mathrm{s})$ \\
\hline 1752 & 8.74 & 10.2 & 1899.3 \\
1789 & 8.31 & 9.3 & 2028.4 \\
1808 & 9.34 & 10.3 & 1889.3 \\
2067 & 10.55 & 6.4 & 1636.6 \\
2165 & 10.47 & 5.2 & 1712.5 \\
2356 & 10.98 & 5.1 & 1986.3 \\
2378 & 10.15 & 3.8 & 2046.3 \\
\hline
\end{tabular}

penetration, we can explain the physical essence of the coefficient $\phi$ : the value of $\phi$ equals the ratio of DOP of an entirely worn projectile to DOP of a rigid projectile with the same mass and impact velocity.

We also notice that the bracket part in equations (38) and (39) equals the DOP of the projectile with the mass of $m_{0}$ at the critical velocity and that the product of the first two terms in equation (40) equals the DOP of jet flow with the length of $L$. Therefore, the physical significance of each term in equations (37)-(39) is clear.

In general, one needs numerous experimental results to determine $\alpha$ and $v_{m}$ by data fitting. However, we find that, in theory, one set of data is sufficient to determine all coefficients.

It follows from equation (37) that

$$
\alpha=\frac{v_{m}}{v_{m}-v_{0}} \ln \frac{m_{\mathrm{res}}}{m_{0}} .
$$

Substituting equation (41) into equation (32) yields the formula for the critical velocity:

$$
v_{m}=\frac{\left(h-h_{f}\right) \beta_{s} \pi r_{0}^{2}+v_{0}\left(m_{0}-m_{\text {res }}\right) / \ln \left(m_{\text {res }} / m_{0}\right)}{m_{\text {res }}+\left(m_{0}-m_{\text {res }}\right) / \ln \left(m_{\text {res }} / m_{0}\right)} .
$$

All parameters in equation (42) are either known or determined by one experiment at hypervelocity. In practice, one usually needs to conduct numerous experiments in a wide velocity range to determine $v_{m}$. With the help of equation (42), however, one can estimate $v_{m}$ by several experiments at arbitrary hypervelocities.

\section{Comparison with Experimental Results}

Li et al. [3] conducted penetration experiments in a wide range of velocities. The experiments were divided into two stages. In the first stage, projectiles with a diameter of $10.8 \mathrm{~mm}$ were ejected towards targets with impact velocities between 1200 and $1810 \mathrm{~m} / \mathrm{s}$. In the second stage, projectiles with a diameter of $7.2 \mathrm{~mm}$ were ejected in the velocity range of $1830-4200 \mathrm{~m} / \mathrm{s}$. Projectiles of the two stages were ogivenose steel rods with a CRH value of 3.0 and a length-diameter ratio of 5. Targets were made of granite and were of the same size in two stages. The photos of posttest projectiles are shown in Figure 8, and the experimental and theoretical DOPs are shown in Figure 9(a) Comparing the photos of projectiles and the curves of DOPs, it is clear that DOPs decrease when dramatic mass loss of projectiles occurs.
Qian et al. [2] conducted penetration experiments with tungsten alloy projectiles and concrete targets. The impact velocities range from 1820 to $3660 \mathrm{~m} / \mathrm{s}$. The projectiles were flat-nose rods, whose diameter was $3.45 \mathrm{~mm}$, and length was $10.5 \mathrm{~mm}$. The experimental and theoretical DOPs are shown in Figure 9(b).

As for metal targets, 6061-T6511 aluminum targets were used by Piekutowski et al. [22]. VAR 4340, 3-CRH-nosed, steel rods were launched at velocities between 550 and $3000 \mathrm{~m} / \mathrm{s}$. The total length of projectiles was $71.1 \mathrm{~mm}$, and the diameter was $7.11 \mathrm{~mm}$. The results for experimental and theoretical DOPs are shown in Figure 9(c).

Forrestal and Piekutowski [1] conducted experiments with the same materials as in Piekutowski et al. [22]. However, the nose of projectiles was spherical rather than ogival. Figure 9(d) shows the experimental and theoretical DOPs of spherical-nose projectiles.

In general, we can see that theoretical results are in good agreement with experimental data. When $v_{0}>v_{m}$, the calculation formula describes well the transition of the projectile from the erosion state to the hydrodynamic state. Comparing Figure 9(c) with Figure 9(d), we can infer that the nose shape of projectiles has effects on the value of $v_{m}$. Blunter nose means larger resistance force acting on projectiles, which naturally results in more dramatic interactions and, consequently, smaller $v_{m}$ for DOP decreasing. The calculated DOPs are kind of smaller than experimental DOPs in Figure 9(d), when the velocity is in the hydrodynamic region. This may be due to the decrease in density of the metal target under high temperature induced by severe interaction.

We can also validate equation (42) against experimental data in Figure 4(a). The initial projectile mass is $32.45 \mathrm{~g}$, and the results are listed in Table 2. The mean value of calculated $v_{m}$ is $1885.5 \mathrm{~m} / \mathrm{s}$, which is quite close to the experimental result, $1600 \mathrm{~m} / \mathrm{s}$.

\section{Conclusions}

A decrease in DOP as impact velocity increases has been observed in numerous penetration experiments. The decrease in DOP occurs at a critical velocity and, in general, is accompanied by a dramatic erosion of projectile mass, as well as the yielding and blunting of projectiles. To investigate the mechanism of DOP decreasing, we analyzed the relationship between the critical velocity and various factors.

We found that, before reaching the critical velocity, the yielding of projectiles has occurred, and the nose shape of projectiles has come to constant geometry, i.e., sphericity. However, the velocity of dramatic mass loss is nearly equal to the critical velocity of DOP decreasing. Thus, we can infer that the decrease in DOP is the result of the dramatic mass loss of projectiles.

The projectile mass loss is the result of multiple factors, including yielding, melting, and wearing of the projectile. When the impact velocity is relatively low, the mass loss is too slight to reverse the DOP, and the DOP increases as the impact velocity increases. At a critical velocity, however, the severe interaction between the projectile and target violates 
the stability of the projectile body, leading to dramatic mass loss of the projectile and, consequently, the decrease in DOP with impact velocity increasing.

We established a semitheoretical model for mass loss of the projectile and obtained a power law of mass decreasing. Then, a continuous formula for DOP at hypervelocity was derived considering the mass loss of the projectile. We compared the theoretical DOP with experimental results of steel projectiles penetrating different targets, including rock, concrete, and metal. The calculated DOP agrees with experimental results and describes well the transition of the projectile from the erosion state to the hydrodynamic state.

At last, the formula for the critical velocity $v_{m}$ was obtained as a by-product of the solving procedure for DOP. One can predict the range of $v_{m}$ with several experimental results at hypervelocities.

\section{Symbols}

$h$ : Depth of penetration (DOP)

$h^{\prime}$ : Instant DOP during penetration

$h_{s 1}: \quad$ DOP during the erosion stage

$h_{s 2}$ : DOP during the rigid stage

$h_{f}: \quad$ DOP of jet flow

$L: \quad$ Length of the entire projectile

$l$ : Instant length of the entire residual projectile during penetration

$A_{0}: \quad$ The cross-section area of the projectile

$r_{0}$ : Projectile radius

$\psi: \quad$ Caliber-radius-head (CRH) value

$m_{0}: \quad$ Initial mass of the projectile

$m$ : Instant mass of the projectile

$m_{\text {res }}: \quad$ Residual mass of the projectile

$t: \quad$ Time counting from the instant of impact

$v_{0}$ : The impact velocity of the projectile

$v_{L}:$ The particle velocity of the projectile on the interface

$v_{t}$ : The particle velocity of the target on the interface

$v_{y}$ : The minimum impact velocity at which the projectile yields

$v_{m}: \quad$ The critical velocity of decrease in DOP

$v_{p}$ : The instant velocity of the projectile tail

$p: \quad$ Pressure on the interface at the instant of impact

$\rho_{p}: \quad$ The density of the projectile

$\rho_{t}$ : The density of the target

$c_{p}$ : The elastic longitudinal wave velocity of the projectile

$c_{t}$ : The elastic longitudinal wave velocity of the target

$D_{t}: \quad$ The shock wave velocity of the target

$a_{1}$, Coefficients determining $D_{t}$

$a_{2}$ :

$\sigma_{c}:$ Resistance force per unit area on the cross-section of the projectile

$\alpha_{s}$, Coefficients determining $\sigma_{c}$

$\beta_{s}:$

$\sigma_{s}: \quad$ Static compression strength

$\sigma_{y}: \quad$ Dynamic compression strength

$\sigma_{s p}:$ Static compression strength of the projectile

$\sigma_{y p}:$ Dynamic compression strength of the projectile

$K_{c}$ : $\quad$ Fracture toughness of the projectile

$f_{c}$ : $\quad$ Axial compression strength of the target $\tau_{s}: \quad$ Shear strength of the target

$\varepsilon_{0}$ : Limit of the shear strain of the target

$G$ : Shear modulus of the target

$H$ : $\quad$ Dynamic hardness of the target

v: Poisson's ratio of the target

$\kappa: \quad$ Coefficient describing the state of the target

$\alpha$ : Coefficient of mass loss

$\lambda_{c}: \quad$ Decrease coefficient

$\vartheta$ : $\quad$ Geometry coefficient of the projectile.

\section{Data Availability}

The data used to support the findings of this study are available from the corresponding author upon request.

\section{Conflicts of Interest}

The authors declare that there are no conflicts of interest regarding the publication of this paper.

\section{Acknowledgments}

This research was financed and fully supported by the National Natural Science Foundation of China (51679249 and 12002358), the Natural Science Foundation of Jiangsu Province, China (BK20190570), and the China Postdoctoral Science Foundation (2018M643853), which are greatly appreciated by the authors.

\section{References}

[1] M. J. Forrestal and A. J. Piekutowski, "Penetration experiments with 6061-T6511 aluminum targets and spherical-nose steel projectiles at striking velocities between 0.5 and $3.0 \mathrm{~km} /$ s," International Journal of Impact Engineering, vol. 24, no. 1, pp. 57-67, 2000.

[2] B. Qian, G. Zhou, J. Li et al., "Penetration depth of hypervelocity tungsten alloy projectile penetrating concrete target," Explosion and Shock Waves, vol. 39, Article ID 083301, 2019.

[3] J. Li, M. Wang, Y. Cheng, and Y. Qiu, "Analytical model of hypervelocity penetration into rock," International Journal of Impact Engineering, vol. 122, pp. 384-394, 2018.

[4] S. A. Silling and M. J. Forrestal, "Mass loss from abrasion on ogive-nose steel projectiles that penetrate concrete targets," International Journal of Impact Engineering, vol. 34, no. 11, pp. 1814-1820, 2007.

[5] J. R. Klepaczko and M. L. Hughes, "Scaling of wear in kinetic energy penetrators," International Journal of Impact Engineering, vol. 31, no. 4, pp. 435-459, 2005.

[6] S. E. Jones, J. C. Foster, O. A. Toness, R. J. DeAngelis, and W. K. Rule, "An estimate for mass loss from high velocity steel penetrators," in Proceedings of the ASME PVP-435 Conf Therm Hydraul Probl Sloshing Phenomena, pp. 227-237, Extrem Loads Struct, Vancouver, Canada, August 2002.

[7] L.-L. He, X.-W. Chen, and X. He, "Parametric study on mass loss of penetrators," Acta Mechanica Sinica, vol. 26, no. 4, pp. 585-597, 2010.

[8] T. Xu, M. Wang, and J. Li, "Dynamic hardness of rock materials under strong impact loading," International Journal of Impact Engineering, vol. 140, Article ID 103555, 2020.

[9] E. I. Shemyakin, "Behavior of rocks under dynamic loads," Soviet Mining Science, vol. 2, no. 1, pp. 8-14, 1966. 
[10] M. J. Forrestal, D. J. Frew, S. J. Hanchak, and N. S. Brar, "Penetration of grout and concrete targets with ogive-nose steel projectiles," International Journal of Impact Engineering, vol. 18, no. 5, pp. 465-476, 1996.

[11] D. J. Frew, S. J. Hanchak, M. L. Green, and M. J. Forrestal, "Penetration of concrete targets with ogive-nose steel rods," International Journal of Impact Engineering, vol. 21, no. 6, pp. 489-497, 1998.

[12] A. C. Whiffin, "The use of flat-ended projectiles for determining dynamic yield stress - II. Tests on various metallic materials," Proceedings of the Royal Society of London - Series A: Mathematical and Physical Sciences, vol. 194, no. 1038, pp. 300-322, 1948.

[13] R. N. Davis, A. M. Neely, and S. E. Jones, "Mass loss and blunting during high-speed penetration," Proceedings of the Institution of Mechanical Engineers - Part C: Journal of Mechanical Engineering Science, vol. 218, no. 9, pp. 1053-1062, 2016.

[14] H. Wu, F. Qian, F. Huang, and Y. Wang, "Projectile nose mass abrasion of high-speed penetration into concrete," Advances in Mechanical Engineering, vol. 4, Article ID 296503, 2015.

[15] H. M. Wen, Y. Yang, and T. He, "Effects of abrasion on the penetration of ogival-nosed projectiles into concrete targets," Latin American Journal of Solids and Structures, vol. 7, no. 4, pp. 413-422, 2010.

[16] J. Ning, Z. Li, T. Ma, and X. Xu, "Failure behavior of projectile abrasion during high-speed penetration into concrete," Engineering Failure Analysis, vol. 115, Article ID 104634, 2020.

[17] H.-W. Yang, X.-C. Jin, J. Zhang, Z.-H. Wang, and Z.-Y. Wang, "Analysis on mass loss of different sized projectiles penetrating into concrete targets," International Journal of Mechanical Sciences, vol. 131-132, pp. 683-692, 2017.

[18] Z. Mu and W. Zhang, "An investigation on mass loss of ogival projectiles penetrating concrete targets," International Journal of Impact Engineering, vol. 38, no. 8-9, pp. 770-778, 2011.

[19] X. W. Chen, L. L. He, and S. Q. Yang, "Modeling on mass abrasion of kinetic energy penetrator," European Journal of Mechanics - A: Solids, vol. 29, no. 1, pp. 7-17, 2010.

[20] G. Li, C. Song, Y. Qiu, and M. Wang, "Theoretical and experimental studies on the phenomenon of reduction in penetration depth of hyper-velocity projectiles into granite," Chinese Journal of Rock Mechanics and Engineering, vol. 37, pp. 60-66, 2018.

[21] M. E. Backman and W. Goldsmith, "The mechanics of penetration of projectiles into targets," International Journal of Engineering Science, vol. 16, no. 1, pp. 1-99, 1978.

[22] A. J. Piekutowski, M. J. Forrestal, K. L. Poormon, and T. L. Warren, "Penetration of 6061-T6511 aluminum targets by ogive-nose steel projectiles with striking velocities between 0.5 and $3.0 \mathrm{~km} / \mathrm{s}$," International Journal of Impact Engineering, vol. 23, no. 1, pp. 723-734, 1999. 